\title{
Cost comparison of predictive genetic testing versus conventional clinical screening for familial adenomatous polyposis
}

\author{
B Bapat, H Noorani, Z Cohen, T Berk, A Mitri, B Gallie, K Pritzker, S Gallinger, \\ A S Detsky
}

Familial GI Cancer Registry, Mount Sinai Hospital, University of Toronto, Toronto, Canada

B Bapat

H Noorani

Z Cohen

T Berk

$S$ Gallinger

Department of Pathology and

Laboratory Medicine, Mount Sinai Hospital, University of Toronto, Toronto, Canada A Mitri

K Pritzker

B Bapat

Department of

Medicine, Mount Sinai Hospital, University of

Toronto, Toronto,

Canada

A S Detsky

Molecular and Medical Genetics, Department of Ophthalmology and Division of

Immunology and

Cancer Research, The

Hospital for Sick

Children and Eye

Research Institute of

Canada, University of

Toronto, Toronto,

Canada

B Gallie

Correspondence to: Dr B Bapat, Department of Pathology and Laboratory

Medicine, Mount Sinai

Hospital, 600 University

Avenue, Toronto, Ontario,

M5G 1X5, Canada.

Accepted for publication

4 November 1998

\begin{abstract}
Background-Mutations of the APC gene cause familial adenomatous polyposis (FAP), a hereditary colorectal cancer predisposition syndrome.

Aims-To conduct a cost comparison analysis of predictive genetic testing versus conventional clinical screening for individuals at risk of inheriting FAP, using the perspective of a third party payer.

Methods-All direct health care costs for both screening strategies were measured according to time and motion, and the expected costs evaluated using a decision analysis model.

Results-The baseline analysis predicted that screening a prototype FAP family would cost $\$ 4975 / £ 3109$ by molecular testing and $\$ 8031 / £ 5019$ by clinical screening strategy, when family members were monitored with the same frequency of clinical surveillance (every two to three years). Sensitivity analyses revealed that the genetic testing approach is cost saving for key variables including the kindred size, the age of screening onset, and the cost of mutation identification in a proband. However, if the $A P C$ mutation carriers were monitored at an increased (annual) frequency, the cost of the genetic screening strategy increased to \$7483/ $£ 4677$ and was especially sensitive to variability in age of onset of screening, family size, and cost of genetic testing of at risk relatives.
\end{abstract}

Conclusions-In FAP kindreds, a predictive genetic testing strategy costs less than conventional clinical screening, provided that the frequency of surveillance is identical using either strategy. An additional significant benefit is the elimination of unnecessary colonic examinations for those family members found to be noncarriers.

(Gut 1999;44:698-703)

Keywords: familial adenomatous polyposis;

adenomatous polyposis coli gene; cost analysis; genetic testing

In recent years, the genes responsible for a number of adult onset hereditary disorders, including breast and colon cancer and Alzheimer's disease, have been identified. The cloning of these disease genes, coupled with sensitive and reliable genetic mutation charac- terisation techniques, has made it possible to identify individuals who have inherited a high risk of developing disease later in life. Predictive genetic testing is thus opening a whole new era of medicine where individuals in high risk families can be screened and counselled before they develop disease. Presymptomatic DNA testing also offers the opportunity for disease prevention by identifying individuals with elevated risk who can benefit from improved surveillance regimens. The potential for cost savings and clinical benefits is significant, particularly as the cost of DNA testing decreases with improvements in the technology.

In this study, we have conducted a cost comparison analysis of predictive DNA testing versus conventional clinical screening for individuals with a family history of familial adenomatous polyposis (FAP). This rare, hereditary, preneoplastic syndrome occurs with a population frequency of one in $10000 .^{12}$ The hallmark of FAP is the development of multiple colorectal adenomatous polyps, typically from the age of puberty onwards. An attenuated variant form, known as AAPC, is characterised by either a later age of onset and/or less florid polyposis. The adenomatous polyps progress to malignancy over a variable time period of $5-10$ years. ${ }^{34} \mathrm{FAP}$ is an autosomal dominant condition associated with a high penetrance and first degree relatives of affected individuals have a $50 \%$ risk of inheriting the disease. ${ }^{45}$ Prior to the advent of predictive genetic testing, screening for the appearance of multiple colorectal adenomatous polyps has been the most effective method to identify high risk members of FAP families. Such conventional screening regimens involve frequent colonic examinations by either flexible sigmoidoscopy or colonoscopy, repeated at regular time intervals. Guidelines for screening asymptomatic patients were established by an international consortium which recommended initiation of flexible sigmoidoscopy from age 10-14 years, repeated every two years until age 40 , and every three to five years thereafter until age $60 .{ }^{1}$

In 1991, the genetic defect responsible for FAP was identified and shown to be due to mutations in the adenomatous polyposis coli $(A P C)$ gene, a tumour suppressor gene that

Abbreviations used in this paper: APC, adenomatous polyposis coli; FAP, familial adenomatous polyposis; HDA, heteroduplex analysis; PTT, protein truncation test; HNPCC, hereditary non-polyposis colorectal cancer. 
maps to chromosome $5 .^{6-9}$ With the cloning of the $A P C$ gene and the high degree of penetrance of germline $A P C$ mutations in FAP families, presymptomatic first degree relatives can now be tested once a germline $A P C$ mutation is identified in an affected FAP patient (the proband). More than $90 \%$ of known germline $A P C$ mutations result in a truncated protein. ${ }^{10}{ }^{11}$ Of these, approximately $18 \%$ of all germline $A P C$ mutations occur at the two mutation hot spot regions, at $A P C$ codons 1061-1063 and 1309-1311. ${ }^{11}$ Since 1992, a predictive molecular genetic screening programme for FAP has been established at the Familial Gastrointestinal Cancer Registry, Mount Sinai Hospital (MSH), Toronto. The aim of this screening programme is to identify germline $A P C$ mutation carriers among first degree relatives of FAP patients prior to the manifestation of clinical symptoms. Major clinical benefits of the genetic screening programme are twofold. FAP family members identified at an increased inherited risk continue with the usual protocol of clinical screening (periodic flexible sigmoidoscopy) while eliminating unnecessary endoscopies for individuals tested negative for the mutation. Because of the reduction in the diagnostic uncertainty, predictive genetic screening strategy is expected to lead to overall better health outcomes for FAP families. ${ }^{12}$

Using the perspective of a third party payer, we carried out a comparison of the costs of predictive genetic testing for $A P C$ mutations versus costs of conventional colorectal screening to detect adenomatous polyps without the benefit of molecular testing. We measured and valued all direct health care costs through detailed monitoring and analysed the data using a decision model. ${ }^{13-16}$

\section{Methods}

PATIENT ACCRUAL

Patients with FAP and their families were identified through a familial Gastrointestinal Cancer Registry located at the Mount Sinai Hospital, University of Toronto. To date, 257 unrelated FAP families have been registered. Patient specimen and data accrual described in this study were carried out according to a protocol approved by the Human Ethics Committee, University of Toronto.

GENETIC TESTING

A genetic testing algorithm has been established in our laboratory and employs initial screening for the two most frequent mutations, at $A P C$ codons $1061-1063$ and $1309-1311$ by heteroduplex analysis (HDA). ${ }^{17}$ If mutations are not detected by this screen, the $A P C$ gene is analysed by protein truncation test (PTT) assay as described previously. ${ }^{18}$ For PTT assay, the entire coding region of the $A P C$ gene is divided into six overlapping segments and sequentially analysed. To date, 124 FAP families have been screened by PTT analysis of the entire coding region of the $A P C$ gene and truncating mutations have been identified in 92 families, indicating a $74 \%(92 / 124)$ sensitivity for the PTT assay.
We evaluated the costs of identifying a germline $A P C$ mutation in blood lymphocytes of FAP patients, using molecular diagnostic technology currently in use in our laboratory. The direct costs for genetic testing included technologists' labour time, data interpretation and reporting by a trained scientific or clinical professional, genetic counselling, laboratory supplies, equipment, and overheads. Sample analysis was based on detailed monitoring of all screen tests. Supplies (for example, chemicals and reagents, disposable) were valued based on the replacement prices and also included an estimate of wastage. All essential laboratory equipment (for example, centrifuges, gel electrophoresis apparatus, etc.) were valued using current replacement costs, on an "annualised" 19 basis using a 5\% discount rate, ${ }^{20}$ and with an assumed working life of five years. Equipment costs per sample were derived by estimating the optimal laboratory caseload. Twenty per cent of total testing costs were allocated to overheads, accounting for laboratory quality assurance services, general utilities, and other operating inputs not identified above, including freight. Two technologists each devote one half of their time to the genetic analysis, and labour costs were based on the technologists' actual gross annual earnings including benefits, and adjusted to account for holiday and sick leave. Molecular diagnostic test design, data interpretation, and reporting were estimated to be one hour for proband and a half an hour for each at risk relative. Genetic counselling was two and a half hours of contact time per proband, and three hours for each at risk relative. Counselling time was based on both direct (for example, pretest explanation, discussion of results) and indirect (phone correspondence, paperwork) communication with each family member, and valued using the (adjusted) salary grade for genetic counsellors.

CLINICAL SCREENING

Each component of the clinical management of FAP families at MSH was observed and associated costs evaluated. These included physician fees, nursing, and clinical coordinator time, other recurrent inputs, a $60 \mathrm{~cm}$ flexible sigmoidoscope, and overheads. A 5\% annual discount rate ${ }^{20}$ was applied to costs for each subsequent procedure following the baseline endoscopy.

Physician fees and the cost for the genetic assessment were derived from the Ontario Ministry of Health Schedule of Benefits. ${ }^{21}$ Nursing (preexamination, examination, and postexamination) and clinical coordinator times were monitored for each patient undergoing flexible sigmoidoscopy. Nursing time was valued using the (adjusted) actual gross annual earnings, and coordinator time was valued based on the (adjusted) salary grade for clinical coordinators.

Other recurrent inputs (for example, medical/surgical supplies) were valued using the General Surgery Clinic budget for the fiscal year. A $60 \mathrm{~cm}$ flexible sigmoidoscope was used for one sixth of an hour per procedure, and valued on an annualised basis, using a five year working life and a $5 \%$ discount rate. Twenty 


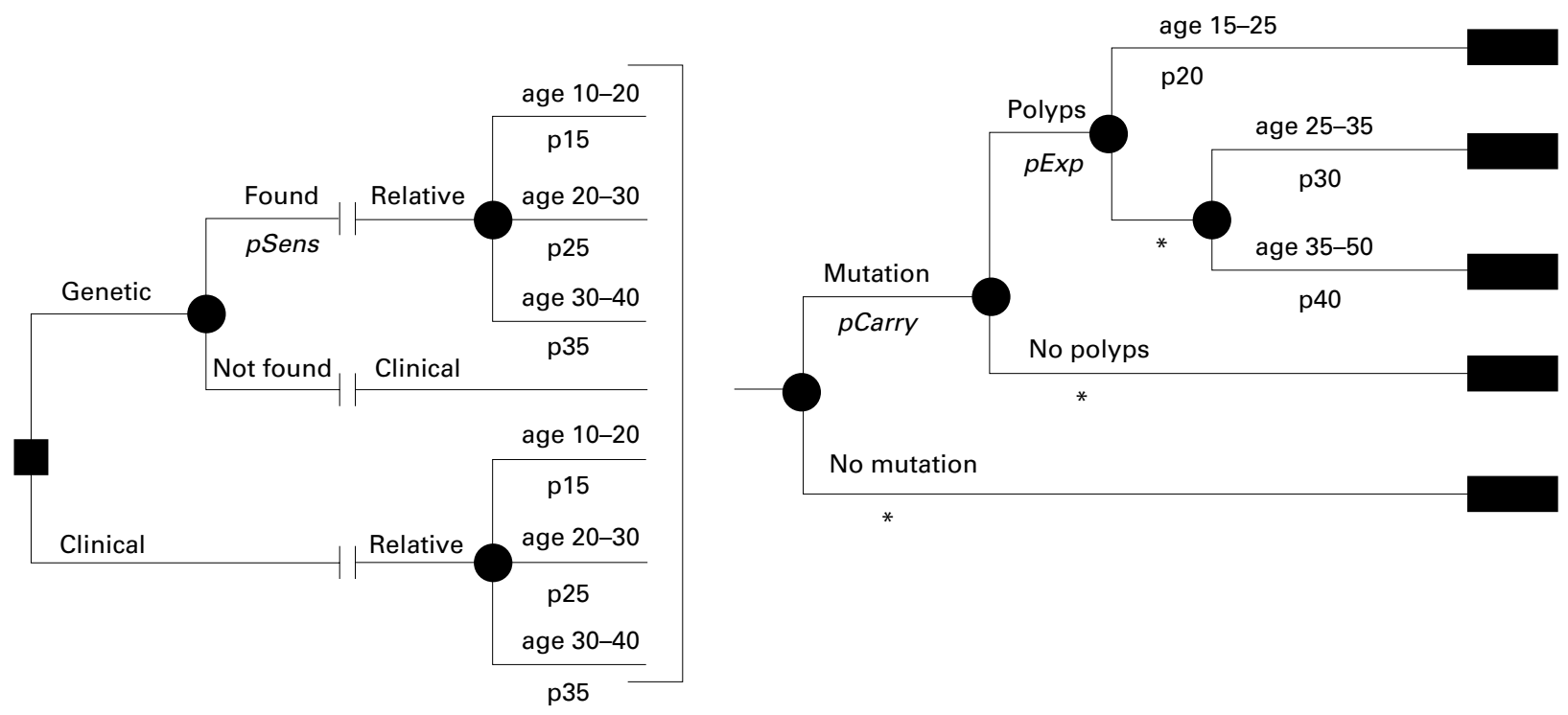

Figure 1 Decision model used in the cost comparison analysis of predictive genetic versus clinical screening strategy. The two options, genetic versus clinical screening, are indicated by the shaded square on the left. Each shaded circle represents chance events; rectangles on the extreme right represent outcomes corresponding to that path in the decision tree; *complementary probability.

per cent of the total costs for each procedure was allocated to overheads, accounting for administrative and support services, utilities, and cost for use of the clinic space.

DECISION ANALYSIS

Baseline analysis

We constructed a decision analysis model to compare genetic and clinical strategies for screening of first degree relatives of FAP patients with the two options (genetic, upper branch; clinical, lower branch) (see fig 1). The circles in the figure represent events that may occur by chance. For the genetic strategy, the initial chance event ( $p$ Sens) is finding or not finding a germline $A P C$ mutation in the proband. If such a mutation is identified, first degree relatives with a $50 \%$ prior inherited risk of polyposis are screened for the presence or absence of the same germline mutation. If the underlying germline $A P C$ mutation is not found in the proband, the first degree relatives are screened using the conventional clinical screening strategy. First degree relatives are classified into three categories with respect to the age of screening onset: $10-20$ years, $20-30$ years, and 30-40 years. For both genetic and clinical strategies, the tree has a similar structure thereafter. Subsequent nodes represent the following chance events: the risk of carrying the $A P C$ mutation ( $p$ Carry); the degree of expression of the $A P C$ mutation

Table 1 Baseline estimates used in the decision model

\begin{tabular}{llll}
\hline Variable & Interpretation & $\begin{array}{c}\text { Baseline } \\
\text { value }\end{array}$ & References \\
\hline$p$ Sens & Sensitivity of genetic strategy for $A P C$ mutation in proband & 0.74 & 18,23 \\
$p 15$ & Proportion of relatives between ages 10 and 20 years & 0.60 & $\dagger$ \\
$p 25$ & Proportion of relatives between ages 20 and 30 years & 0.20 & $\dagger$ \\
$p 35$ & Proportion of relatives between ages 30 and 40 years & 0.20 & $\dagger$ \\
$p$ Carry & Risk of carrying $A P C$ mutation & 0.50 & 1,2 \\
pExp & Degree of expression of $A P C$ mutation & 0.90 & 23 \\
$p 20$ & Detection of FAP polyps between ages 15 and 25 years & 0.90 & $\dagger$ \\
$p 30$ & Detection of FAP polyps between ages 25 and 35 years & 0.99 & $\dagger$ \\
$p 40$ & Detection of FAP polyps between ages 35 and 50 years & 1.00 & $\dagger$ \\
\hline
\end{tabular}

†Authors' estimates.
Table 2 Genetic testing costs

\begin{tabular}{|c|c|}
\hline Sample & Cost/sample $(\$ \mid f)$ \\
\hline \multicolumn{2}{|l|}{ Proband } \\
\hline Tests^ & $207.74 / 129.38$ \\
\hline Equipment & $26.94 / 16.84$ \\
\hline $20 \%$ overhead & $46.94 / 29.34$ \\
\hline Data interpretation/report writing & $49.77 / 31.11$ \\
\hline Genetic counselling & $89.02 / 55.64$ \\
\hline Total & $420.41 / 262.76$ \\
\hline \multicolumn{2}{|l|}{ Relative } \\
\hline Tests* & $89.08 / 55.68$ \\
\hline Equipment & $11.19 / 6.99$ \\
\hline $20 \%$ overhead & $20.05 / 12.53$ \\
\hline Data interpretation/report writing & $24.88 / 15.55$ \\
\hline Genetic counselling & $106.83 / 66.77$ \\
\hline Total & $252.03 / 157.52$ \\
\hline
\end{tabular}

${ }^{\star}$ Cost per test includes technologist labour and supplies.

( $p E x p$ ), which is indicated by the probability of detecting adenomatous polyps characteristic of FAP at ages $15-25$ years $(p 20), 25-35$ years $(p 30)$, and $35-50$ years $(p 40)$. The outcome measures evaluated are direct health care costs. The decision model was constructed and evaluated using the software program SMLTREE. ${ }^{22}$

Seven major assumptions were made in our baseline model: (1) A prototype family consists of one proband and six first degree relatives (range 1-10, table 4) at 50\% inherited risk for FAP, based on enumeration of more than 250 FAP families in the registry. (2) The proband is the first clinically affected FAP patient identified with a germline $A P C$ mutation in a family. (3) The genetic strategy is $74 \%$ sensitive for the detection of an $A P C$ mutation in the proband. (4) In FAP families with a known germline $A P C$ mutation, the genetic strategy is $100 \%$ accurate for screening for the presence or absence of the mutation among first degree relatives. (5) The average age at clinical screening onset is 15 years for $60 \%$ of relatives, 25 years for $20 \%$ of relatives, and 35 years for $20 \%$ of relatives. (6) The time horizon for clinical screening is 40 years (age 15-45 years). (7) The final and most significant assumption concerns the frequency of clinical screening for 
Table 3 Clinical screening costs

\begin{tabular}{llll}
\hline Input & Cost/examination $(\$ / £)$ & $\begin{array}{c}\text { Recommended no } \\
\text { of examinations }\end{array}$ & Cost/patient (\$/\&) \\
\hline $\begin{array}{l}\text { Physician fees } \\
\text { Other personnel }\end{array}$ & $129.55 / 80.97$ & & \\
$\begin{array}{l}\text { Other recurrent/flexible } \\
\quad \text { sigmoidoscopy }\end{array}$ & $69.72 / 43.58$ & & \\
$\begin{array}{l}20 \% \text { overhead } \\
\text { Subtotal }\end{array}$ & $14.42 / 9.01$ & & $2182.35 / 1363.97^{\star}$ \\
Genetic assessment & $256.43 / 160.27$ & 17 & $55.90 / 34.94$ \\
Total & & & $2238.25 / 1398.91$ \\
\hline
\end{tabular}

Patients aged $10-50$ years.

$\star_{5} \%$ discount rate. of subsequent testing of each at risk family member (table 2). Technologist labour and supplies constituted over $50 \%$ of the proband costs.

CLINICAL SCREENING COSTS

At our Registry, conventional clinical screening of first degree relatives of FAP patients consists of a baseline flexible sigmoidoscopy at 10 years of age, and follow up every two years to age 35 and every three to five years to age 50 (17 examinations in total). ${ }^{4}$ These guidelines are in accordance with internationally accepted protocols of the Leeds Castle Polyposis Group. Table 3 shows the direct costs of clinical screening for each at risk relative up to the fifth decade of life. Physician fees make up $50 \%$ of the total costs for each sigmoidoscopic examination. intervals to age 35 , and every three to five years to age 50 for $A P C$ mutation carriers only in the genetic screening strategy (with no sigmoidoscopy for those who do not carry the mutation) and all at risk family members in the clinical screening strategy. Furthermore, in the genetic screening strategy, if a mutation is not found in the proband, all relatives follow the clinical screening strategy. (b) FAP-model B: annual flexible sigmoidoscopy from age of screening onset to age 50 for $A P C$ mutation carriers (again with no sigmoidoscopic screening for non-carriers of $A P C$ mutations), while sigmoidoscopic screening frequency is unchanged for the arm that was not genetically screened (fig 1). Table 1 summarises the baseline probability values used in the decision model.

Sensitivity analysis

All variables of the decision analysis model were examined over a wide range of values in one way sensitivity analyses. The threshold ${ }^{24}$ (that is, the point at which two screening strategies have equal expected costs) was determined for each of the variables in order to evaluate the robustness of the results of the baseline analyses under FAP models A and B as described above.

\section{Results}

GENETIC TESTING COSTS

The direct cost of genetic testing for the proband was almost twofold more than the cost

Table 4 One way sensitivity analyses

\begin{tabular}{|c|c|c|c|c|c|}
\hline \multirow[b]{2}{*}{ Variable } & \multirow[b]{2}{*}{ Interpretation } & \multirow{2}{*}{$\begin{array}{l}\text { Baseline } \\
\text { value }\end{array}$} & \multirow{2}{*}{$\begin{array}{l}\text { Range of } \\
\text { values }\end{array}$} & \multicolumn{2}{|c|}{ Threshold value* } \\
\hline & & & & $A \dagger$ & $B \neq$ \\
\hline pSens & Sensitivity of genetic strategy for $A P C$ mutation in proband & 0.74 & $0.00-1.00$ & 0.10 & 0.31 \\
\hline p15 & Proportion of relatives between ages 10 and 20 years & 0.60 & $0.00-1.00$ & 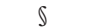 & 0.37 \\
\hline$p 25$ & Proportion of relatives between ages 20 and 30 years & 0.20 & $0.00-1.00$ & ఏ & 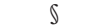 \\
\hline$p 35$ & Proportion of relatives between ages 30 and 40 years & 0.20 & $0.00-1.00$ & d & $\$$ \\
\hline pCarry & Risk of carrying $A P C$ mutation & 0.50 & $0.00-1.00$ & 0.84 & 0.55 \\
\hline$p \operatorname{Exp}$ & Degree of expression of $A P C$ mutation & 0.90 & $0.00-1.00$ & $\int$ & 0.71 \\
\hline$p 20$ & Detection of FAP polyps between ages 15 and 25 years & 0.90 & $0.00-1.00$ & $\sqrt{d}$ & 0.20 \\
\hline$p 30$ & Detection of FAP polyps between ages 25 and 35 years & 0.99 & $0.00-1.00$ & $\$$ & 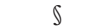 \\
\hline$p 40$ & Detection of FAP polyps between ages 35 and 50 years & 1.00 & $0.00-1.00$ & $\$$ & $\$$ \\
\hline$N$ & Number of relatives in a family & 6 & $1-10$ & 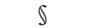 & 2.18 \\
\hline cProb & Cost for genetic testing for $A P C$ mutation in proband & 420 & $100-2500$ & $\S$ & 1154 \\
\hline$c R e l$ & Cost for genetic testing of each relative & 252 & $100-2500$ & 851 & 396 \\
\hline
\end{tabular}

*The threshold ${ }^{19}$ value is a value where the two screening strategies have equal expected costs. If a given variable has a value less than the threshold value, then one strategy is cheaper; if the variable has a value greater than the threshold, then the alternative strategy is cheaper.

†Assuming the same frequency of clinical screening for both strategies (FAP model A).

łAssuming anual screening for relatives testing positive for the $A P C$ mutation under genetic route (FAP model B).

$§$ The genetic strategy is less expensive than the clinical one over all the given range of values (no threshold). 
of at risk relatives is $37 \%$ instead of $60 \%$ for the age of screening onset group 10-20 years; (c) the degree of expression of the $A P C$ mutation, defined as the probability of occurrence of adenomatous polyps, decreases to 0.71 ; and $(d)$ the probability of detection of adenomatous polyps between ages 15 and 25 years falls from 0.90 to 0.20 .

\section{Discussion}

We performed a detailed comparison of all direct health care costs for genetic or conventional clinical screening for FAP. The predictive genetic testing approach costs about one third to one thirteenth less than that of the conventional clinical strategy over a wide range of variables. Thus, on the basis of economic variables alone, molecular genetic testing was the method of choice. A significant finding of this study was that the genetic testing strategy saved substantial costs only if the sigmoidoscopic surveillance regimens for $A P C$ mutation carriers remained the same as for conventional clinical screening. FAP is a paradigm for cancer prevention based on the known adenoma-carcinoma sequence which occurs over an average of 5-10 years. Prophylactic colectomy in affected individuals essentially eliminates risk of colon cancer, although individuals may still be at risk of rectal cancer depending on the specific prophylactic operation performed and subsequent screening of the remaining rectum, and for other extracolonic manifestations associated with FAP. Clinical screening regimens have been established to ensure that at risk offspring and siblings of FAP patients benefit from early diagnosis and treatment. Interestingly, several registries have now advocated annual flexible sigmoidoscopy for presymptomatic family members known to carry an APC mutation..$^{25-27}$ The efficacy of increasing surveillance frequency from a biennial to annual time interval for $A P C$ mutation carriers is not yet proved, especially in the absence of histological or clinical data to support such findings. Early studies by Morson (1974) found an average $5 \%$ malignancy rate for adenomas over a life span and showed that only a few adenomas in FAP ever become malignant. ${ }^{3}$ The minimal interval of five years for such a transition would suggest the lack of an accelerated evolutionary process, such as that indicated for colorectal adenomas associated with hereditary nonpolyposis colorectal cancer (HNPCC). ${ }^{28}$

The decision analysis carried out in this study was conservative for several reasons. Firstly, if a germline $A P C$ mutation is not detected by HDA and/or PTT assay, molecular linkage analysis using intragenic and closely linked polymorphic DNA markers can achieve predictive carrier risk estimates with greater than $99 \%$ accuracy in informative families. ${ }^{5930}$ For families with a suitable pedigree structure, the inclusion of linkage based testing would result in greater cost savings under the genetic route. Several different molecular diagnostic techniques can be used for predictive genetic testing of FAP. ${ }^{69}{ }^{17}{ }^{23}$ The choice of optimal technique(s) is largely dependent on the nature of mutations and the frequency of specific mutations. We chose PTT for mutation analysis as the majority of germline $A P C$ mutations are truncating in nature. Another assumption regarding the time span for conventional clinical screening is also conservative as at risk relatives continue to be examined after age 50 years, albeit at a low frequency. ${ }^{4}$ Given, however, that the costs for each subsequent procedure following the baseline endoscopy were discounted at a rate of $5 \%$ per year to their present values, extension of the screening time frame would not significantly affect our analytical result. Finally, the cost comparison analysis was done with the perspective of a third party payer, and therefore, only the costs directly related to the comparative strategies were considered. Inclusion of direct personal and indirect costs, such as lost productivity of patients and accompanying family members during each clinic visit for a sigmoidoscopic examination, would result in further cost savings under the genetic route.

What are the implications of such a cost analysis for screening members of FAP families? Apart from the lower cost, the genetic route is less invasive, needs to be performed only once, and can be carried out early in life, thereby significantly modifying the $50 \%$ inherent risk of FAP in at risk relatives. ${ }^{45}$ In individuals identified as high risk, surveillence regimens can also be initiated for extracolonic manifestations such as upper gastrointestinal polyps and cancer. Because the age of onset of FAP adenomas is variable, patient compliance with screening regimens remains an important factor for the optimal management of this disorder. On the other hand, a reduced genetic risk will relieve anxiety associated with frequent colonic examination ${ }^{531}$; clinicians can now focus on those asymptomatic patients identified as "high risk" by genetic testing, but yet for whom one cannot predict the clinical sequelae. More importantly, family members identified to be non-mutation carriers can be released from unnecessary clinical surveillance. From the analysis presented here, we conclude that substantial direct cost savings could result from the adoption of a genetic screening strategy for FAP, but only if the frequency of sigmoidoscopic screening is not increased for asymptomatic patients carrying the $A P C$ mutation. Given the limited health care resources available, not only to screen and treat but also to counsel affected FAP families, it would seem prudent to remain focused on a biennial screening regimen.

The authors would like to thank Dr Alan Bernstein for his support and helpful suggestions throughout this project, and Dr Carol Swallow for critical reading of the manuscript. We are this study. This work was supported in part by funds from Canadian Genetic Diseases Network (HN, BG).

1 Bulow S, Burn J, Neale K, et al. The establishment of a polyposis register. Int $\mathcal{F}$ Colorectal Dis 1993;8:34-8.

2 Bussey HJR. Familial polyposis coli. Family studies, histopathology, differential diagnosis, and results of treatment. Baltimore: ogy, differential diagnosis, and results of treat
Johns Hopkins University Press, 1975.

3 Morson B. The polyp-cancer sequence in the large bowel. Proc R Soc Med 1974;67:451-7.

4 Berk T, Stern HS. Screening, management, and surveillance for families with familial adenomatous polyposis. Gastrointest Endosc Clin North Am 1993;3:725-36. 
5 Bapat BV, Parker JA, Berk T, et al. Combined use of molecular and biomarkers for presymptomatic carrier risk familial adenomatous polyposis: implications for screening guidelines. Dis Colon Rectum 1994;37:165-

6 Groden J, Thliveris A, Samowitz W, et al. Identification and characterization of the familial adenomatous polyposis coli gene. Cell 1991;66:589-600

7 Kinzler KW, Nilbert MC, Su LK, et al. Identification of FAP locus genes from chromosome 5q21. Science 1991;253: $661-5$.

8 Joslyn G, Carlson M, Thliveris A, et al. Identification of deletion mutations and three new genes at the familial polyposis locus. Cell 1991;66:601-13.

9 Nishisho I, Nakamura Y, Miyoshi Y, et al. Mutations of chromosome 5q21 genes in FAP and colorectal cancer patients. Science 1991;253:665-9.

10 Nagase H, Nakamura Y. Mutations of the APC (adenomatous polyposis coli) gene. Hum Mutat 1993;2:425-34.

11 Beroud C, Soussi T. APC gene: data base of germline and somatic mutations in human tumors and cell lines. Nucleic Acids Res 1996;24:121-4.

12 Belchetz LA, Berk T, Bapat B, et al. Changing causes of mortality in patients with familial adenomatous polyposis. Dis Colon Rectum 1996;39:384-7.

13 Sox HC, Blatt MA, Higgins MC, et al. Medical decision making. Stoneham: Butterworths, 1988

14 Kassirer JP, Moskowitz AJ, Lau J, et al. Decision analysis: a progress report. Ann Intern Med 1987;106:275-91.

15 Richardson WS, Detsky AS, for the Evidence-Based Medicine Working Group. Users' guides to the medical literature. VII. How to use a clinical decision analysis. A Are the results of the study valid? $7 A M A 1995 ; 273: 1292-5$.

16 Richardson WS, Detsky AS, for the Evidence-Based Medicine Working Group. Users' guides to the medical literature. VII. How to use a clinical decision analysis. B. What are the results and will they help me in caring for my patients? fAMA 1995;273:1610-13.

17 Mandl M, Paffenholz R, Friedl W, et al. Frequency of common and novel inactivating APC mutations in 202 families mon and novel inactivating APC mutations in 202 families
with familial adenomatous polyposis. Hum Mol Genet with familial
18 Powell SM, Peterson GM, Krush AJ, et al. Molecular diagnosis of familial adenomatous polyposis. $N$ Engl $7 \mathrm{Med}$ .

19 Cleverley WO. Capital Budgeting. In: Cleverley WO, ed. Handbook of health care accounting and finance. 2nd edn. Rockville: Aspen, 1989:285-310.

$20 \mathrm{Krahn}$ M, Gafni A. Discounting in the economic evaluation of health care services. Med Care 1993;31:403-18.

21 Schedule of Benefits: physician services under the Health Insurance Act. Ontario Ministry of Health, 1992.

22 Hollenberg JP. SMLTREE, Version 2.9. Roslyn, NY, 1989.

23 Prosser J, Condie A, Wright M, et al. APC mutation analysis by chemical cleavage of mismatch and a protein truncation assay in familial adenomatous polyposis. Br f Cancer 1994; 61:841-6.

24 Pauker SG, Kassirer JP. The threshold approach to clinical decision making. $N$ Engl f Med 1980;302:1109-17.

25 Burt R, Groden J. The genetic and molecular diagnosis of adenomatous polyposis coli. Gastroenterology 1993;104: 1211-14.

26 Petersen GM. Knowledge of the adenomatous polyposis coli gene and its clinical application. Ann Med 1994;26: coli gen

27 Park J-G, Han H-J, Won Y-J, et al. Familial adenomatous polyposis and hereditary non-polyposis colorectal cancer in Korea. In: Baba S, ed. New strategies for treatment of hereditary colorectal cancer. Tokyo: Churchill Livingstone, 1996: 213-28.

28 Jass JR, Stewart SM. Evolution of hereditary non-polyposis colorectal cancer. Gut 1992;33:783-6.

29 MacDonald F, Morton DG, Rindl PM, et al. Predictive diagnosis of familial adenomatous polyposis with linked DNA markers: a population based study. BMF 1992;304: 869-72.

30 Burn J, Chapman P, Delhanty JDA, et al. The UK Northern Region genetic register for familial adenomatous polyposis coli: the use of age of onset, congenital hypertrophy of the coli: the use of age of onset, congenital hypertrophy of the
retinal pigment epithelium, and DNA markers in risk retinal pigment epithelium, and DNA mar
calculations. $\mathcal{F}$ Med Genet 1991;28:289-96.

31 Calculations. F Med Genet 1991;28:289-96. agement of familial adenomatous polyposis. Gut 1992;33: agement $125-31$. 